\title{
Pericarditis during chronic haemodialysis therapy
}

\author{
A. G. Mitchell \\ B.A., B.M., B.CH., M.R.C.P. \\ Artificial Kidney Unit, University Hospital of South Manchester, \\ Withington Hospital, Manchester
}

\begin{abstract}
Summary
Pericardial disease in a haemodialysis unit has been reviewed. It is suggested that in addition to the well established uraemic pericarditis there is a pericarditis with a different clinical course occurring in patients on chronic haemodialysis and with an incidence in this series of $18 \%$.
\end{abstract}

\section{Introduction}

Pericarditis is a well established complication of uraemia and has a grave prognosis. The development of dialysis and renal transplantation has transformed this prognosis but regular dialysis does not entirely prevent the development of pericarditis (Beaudry, Nakamoto and Kolff, 1966; Bailey et al., 1968; Comty, Cohen and Shapiro, 1971).

This study reviews pericardial disease in a haemodialysis centre over the 5-year period from January 1968 to December 1972 in order to assess the course of the disease and attempt to identify the factors involved in the development of the pericarditis.

\section{Patients}

Eighty-three patients (fifty male, thirty-three female) were accepted for chronic haemodialysis during the period of this study. Haemodialysis was performed on a regular basis using a Scribner shunt or latterly a Brescia fistula for three $10-\mathrm{hr}$ periods/ week at a blood flow rate of $180-200 \mathrm{ml} / \mathrm{min}$ on a modified Kiil kidney. A continuous heparin infusion was used and protamine was not routinely given. This was supplemented by a $60 \mathrm{~g}$ protein diet with restriction of salt and water in order to keep the patients normotensive and free from oedema.

All patients came to the unit after a variable period of conservative management on a modified Giovanetti diet and intermittent peritoneal dialysis. Three patients had pericarditis at the onset of chronic haemodialysis. After an initial period of stabilization by haemodialysis it was rare to detect the neuromuscular, gastro-intestinal or haemorrhagic features seen so commonly in severe uraemia unless problems of dietary control occurred.

Present address: Division of Cardiovascular Disease, Hammersmith Hospital, Du Cane Road, London, W.12.
In all cases but one, the diagnosis of pericarditis rested on the presence of a pericardial friction rub. The exception had pericardial disease discovered only at post mortem. During the course of clinical pericarditis, chest radiology and electrocardiograms were performed when clinically indicated.

\section{Results}

After the establishment of regular haemodialysis therapy, clinical pericarditis was found in fifteen patients, while one further patient was found to have an unsuspected serous pericardial effusion at autopsy. These sixteen patients comprised nine males and seven females. Their age distribution is shown in Fig. 1 compared with the age distribution of all patients at the end of the period of observation or at death.

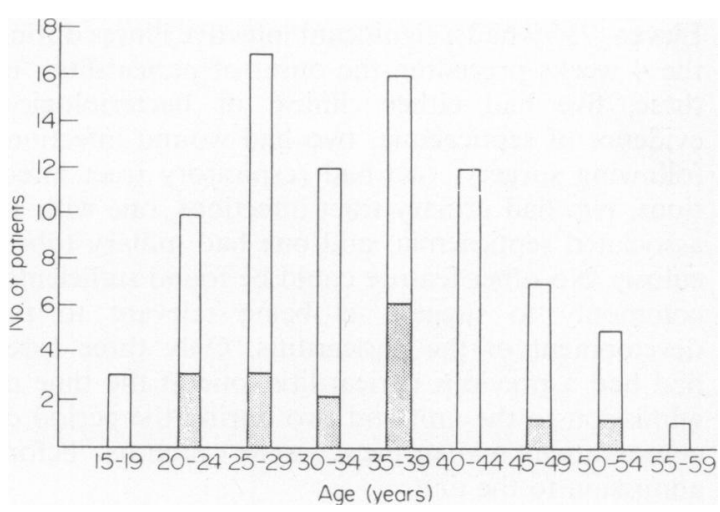

FIG. 1. Age distribution of patients. Blank, all patients; stippled, patients with pericarditis.

Seven patients with pericardial disease subsequently died: a patient who died suddenly at home was the one who had an unsuspected serous pericardial effusion and also had severe coronary artery disease. In a second patient, known to have miliary tuberculosis and pericarditis, autopsy showed a haemorrhagic pericardial effusion but there was no histological evidence of tuberculosis in the pericardium. In the remaining five, no relationship could 


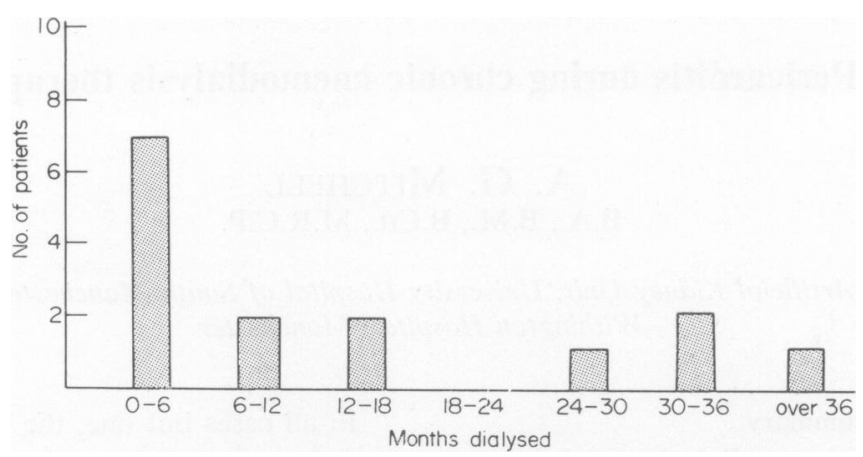

FIG. 2. Duration of dialysis in pericarditis patients.

be established between the pericarditis and the presumed cause of death. Two cases showed pericardial adhesions, one with fibrous obliteration of the whole pericardial cavity 8 months after the pericarditis had resolved clinically. The second case showed fibrinous adhesions 2 months after the pericarditis had resolved. Information from autopsy was not available in three cases.

The relationship between the development of pericarditis and the duration of haemodialysis is seen in Fig. 2.

\section{Clinical features}

Fifteen patients developed pericarditis after regular haemodialysis therapy had been established. Eleven $(73 \%)$ had a significant infective illness during the 4 weeks preceding the onset of pericarditis; of these, five had either clinical or bacteriological evidence of septicaemia, two had wound infections following surgery, two had respiratory tract infections, two had urinary tract infections, one with an associated septicaemia, and one had miliary tuberculosis. No other feature could be found sufficiently commonly to suggest its being relevant to the development of the pericarditis. Only three cases had had a previous pericarditis, one at the time of admission to the unit and two during the period of conservative management some months before admission to the unit.

\section{Symptoms}

In five $(33 \%)$ of the fifteen cases of pericarditis the onset was asymptomatic, and noted during routine examination. The symptoms in the remaining patients were chest pain in seven $(47 \%)$, nonproductive cough in six $(40 \%)$, dyspnoea in six $(40 \%)$, and malaise in ten $(66 \%)$.

\section{Signs}

Fourteen $(93 \%)$ cases had a pyrexia on presentation and this often persisted throughout the duration of the pericarditis. Sinus tachycardia was common and two patients developed multiple supraventricular ectopics and two developed atrial fibrillation. The pericardial friction rub persisted between 3 days and 10 weeks (mean duration 25 days). In only three patients did it last under 1 week and in ten cases it persisted for over 4 weeks. In thirteen $(86 \%)$ a degree of cardiomegaly developed and a gallop rhythm was present in ten $(66 \%)$. Nine $(60 \%)$ developed significant cardiac failure as shown by elevation of the jugular venous pressure, and/oro left ventricular failure. Loss of body weight occurred in six $(40 \%)$ cases ranging up to $8.5 \mathrm{~kg}$. Clinical or radiological evidence of a pericardial effusion occurred in seven cases and four proceeded to develop cardiac tamponade with a paradoxical pulse, tachycardia, hypotension, and elevation of jugular venous pressure. Clinical pulmonary changes occurred in five (33\%) patients. One developed left lower lobe consolidation, three developed pleural effusions and one had widespread crepitations from miliary tuberculosis.

\section{Investigations}

Table 1 shows the plasma urea of thirteen patients and the plasma creatinine of five patients with pericarditis. These values were taken after the onset of pericarditis and before the next haemodialysis. The plasma urea exceeded $150 \mathrm{mg} / 100 \mathrm{ml}$ in only four of these patients and only one was in excess of 200 . The table also shows the pre-dialysis urea and creatinine values of twenty consecutive patients without pericarditis at the time of their admission to the unit and after a period of regular haemodialysis therapy.

A significant leucocytosis developed in six $(40 \%)$ cases but was usually mild and late in the course of the disease. The sera of four patients were examined for fibrin degradation products and two for myocardial antibodies but no significant abnormalities were revealed. Liver function tests were obtained in nine patients but did not reveal any consistent abnormality. 
TABle 1. Plasma urea of thirteen patients and plasma creatinine of five patients with pericarditis.

\begin{tabular}{|c|c|c|}
\hline & $\begin{array}{l}\text { Plasma urea } \\
(\mathrm{mg} / 100 \mathrm{ml})\end{array}$ & $\begin{array}{l}\text { Plasma creatinine } \\
(\mathrm{mg} / 100 \mathrm{ml})\end{array}$ \\
\hline $\begin{array}{l}\text { Controls } \\
\text { Before chronic haemodialysis } \\
\text { After chronic haemodialysis }\end{array}$ & $\begin{array}{c}\text { Mean } 200 \\
\text { Range } 120-300 \\
\text { Mean } 135 \\
\text { Range } 80-175\end{array}$ & $\begin{array}{c}\text { All over } 13 \cdot 5 \\
60 \% \text { greatly over } 15 \\
\text { Mean } 11 \cdot 3 \\
\text { Range } 8 \cdot 5-14 \cdot 1\end{array}$ \\
\hline Pericarditis patients & $\begin{array}{c}\text { Mean } 145 \\
\text { Range } 80-210 \\
\text { (thirteen patients) }\end{array}$ & $\begin{array}{c}\text { Mean } 10.9 \\
\text { Range } 4 \cdot 3-15 \cdot 8 \\
\text { (five patients) }\end{array}$ \\
\hline
\end{tabular}

Bacterial isolation from skin, nose and throat was attempted on all patients and blood cultures were obtained from all pyrexial patients. Sputum, urine, wound swabs, pericardial fluid and dialysis fluid were cultured when clinically indicated. Viral serology and attempts at isolation from throat and faeces were performed in a small number of patients. No consistent abnormalities were found except for the presence of a bacterial infection during the month preceding the pericarditis. All specimens of pericardial fluid obtained were sterile.

ECG changes were noted in eleven ( $73 \%$ ) cases, three $(20 \%)$ having abnormal ST elevation and T wave changes characteristic of pericarditis, while eight $(54 \%)$ showed non-specific $T$ wave changes. All these changes subsequently reversed.

Repeated chest radiology during the course of the pericarditis was performed in twelve patients, all of whom had clinical pericarditis for at least 7 days. For periods shorter than this radiological changes could not definitely be related to the pericarditis although one case showed evidence of miliary tuberculosis. All twelve showed a marked increase of the cardiothoracic ratio with return to the initial heart size or less as the illness resolved. Six of these patients developed the classical configuration of a pericardial effusion and all except one of these was proven by screening or aspiration. A pleural effusion occurred in six patients but generally was associated with inflammatory or congestive changes.

\section{Management}

During the course of the pericarditis haemodialysis was maintained with whole body heparinization in all except five patients. One patient received several peritoneal dialyses because of difficulty with the Brescia fistula. The other four received a total of eight peritoneal dialyses in an attempt to avoid heparinization in the presence of the pericarditis. When peritoneal dialysis was employed patients were given a Giovanetti diet.

The onset of pericarditis could not be related to the procedure of haemodialysis since the onset of symptoms occurred both during and after completion of dialysis. In a number of patients with a pyrexia, haemodialysis led to a temporary exacerbation of the fever requiring premature termination of the dialysis. No other adverse effects could be attributed directly to haemodialysis although the development of a pericardial effusion was usually insidious. Of the four patients who developed pericardial tamponade, three were being maintained on haemodialysis while the fourth had been maintained on peritoneal dialysis for the previous 2 weeks. One patient treated by peritoneal dialysis retained fluid with a weight gain of $9 \mathrm{~kg}$ and this precipitated severe cardiac failure.

Cardiac failure was treated by removal of fluid during dialysis and three patients also received digitalis therapy. Digitalis was also given to two patients who developed atrial fibrillation. In one case the arrhythmia was transient but caused marked impairment of the Brescia fistula flow, while the other case persisted for 3 months before reverting to sinus rhythm $24 \mathrm{hr}$ after starting quinidine therapy. All patients have subsequently had digitalis withdrawn. Four patients have had pericardial aspiration performed. Three patients were aspirated for pericardial tamponade with the removal of between 150 and $1250 \mathrm{ml}$ of fluid. A fourth patient received a pericardial tap to exclude a pyogenic pericarditis but no effusion was detected. In all cases pericardial fluid was heavily blood-stained.

Antibiotics were used in nine patients to treat known infections according to the nature and source of that infection. In no case did antibiotics produce any effect on the development and course of the pericarditis.

\section{Case histories}

Two case histories are given to demonstrate the pericarditis seen in this series.

\section{Case 1}

An 18-year-old male, with chronic glomerulonephritis proceeding to chronic renal failure.

Started regular haemodialysis each day via a 
subclavian vein cannula, then three times weekly via a Brescia fistula. Admission to this unit followed a period of several months in terminal renal failure. Pericarditis developed 3 days before the onset of haemodialysis (blood urea $164 \mathrm{mg} / 100 \mathrm{ml}$, creatinine $>15 \mathrm{mg} / 100 \mathrm{ml}$ ) and was accompanied by severe malaise, epistaxes, uraemic asterexis and twitching, and an early peripheral neuropathy. No chest pain occurred but a prominent pericardial friction rub developed and persisted for only 3 days after regular haemodialysis was instituted. Simultaneously, the neurological abnormalities showed marked improvement. There was no pyrexia associated with the pericarditis. Follow up over 11 months has revealed no cardiovascular complications.

\section{Case 2}

A 30-year-old female with chronic pyelonephritis proceeding to chronic renal failure.

Started regular haemodialysis three times weekly via Brescia fistula. There was no evidence of pericarditis before onset of haemodialysis and haemodialysis was performed for 16 months before pericarditis developed.

Twenty-seven months after starting regular haemodialysis she developed a rigor on haemodialysis $\left(\mathrm{T}=101^{\circ} \mathrm{F}\right)$ which did not recur. This was associated with a high bacterial count in the dialysate. Two weeks later she developed generalized myalgia, with a sore throat and became pyrexial. $(\mathrm{Hb}=6.3 \mathrm{~g} / 100 \mathrm{ml}, \mathrm{WCC}=13,000$, throat swab yielded normal flora.) Six days later she presented with precordial chest pain and general malaise. Examination revealed a pyrexia $\left(101^{\circ} \mathrm{F}\right)$, bilateral basal crepitations and a loud pericardial friction rub. (Blood urea $173 \mathrm{mg} \%$, potassium $4.5 \mathrm{mEq} / \mathrm{l}, \mathrm{Hb}$ $5 \cdot 3 \mathrm{~g} / 100 \mathrm{ml}$, WCC 15,500 , reticulocytes $1 \%$.) Management commenced with the institution of a rigid Giovanetti diet and peritoneal dialysis was performed. The pyrexia persisted for 4 weeks in spite of cloxacillin. The pericardial friction rub persisted for 4 weeks. During this time two episodes of rapid atrial fibrillation occurred precipitating marked cardiac failure. At this time there was no evidence of cardiac tamponade and the fibrillation was controlled by digitalis. After 3 weeks a rapid deterioration occurred with dyspnoea, marked elevation of jugular venous pressure, falling blood pressure and diminished cardiac pulsations. Pericardial aspiration of $900 \mathrm{ml}$ haemorrhagic fluid gave marked improvement. Over a 31-day period four peritoneal dialyses were performed maintaining the blood urea below $200 \mathrm{mg} \%$. Following the disappearance of the rub haemodialysis was restarted. Serial ECGs showed transient $T$ wave depression over the inferior limb leads and V3-6. There was an overall weight loss of $6 \mathrm{~kg}$. Routine bacteriology revealed no abnormalities.
The haemoglobin fell during the pericarditis $(\mathrm{Hb}$ $6.4 \mathrm{~g} / 100 \mathrm{ml}$ to $4.4 \mathrm{~g} / 100 \mathrm{ml}$ ) rising again after

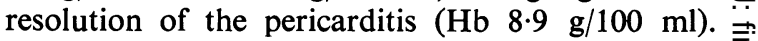
Follow up after 16 months has revealed no cardiovascular complications.

\section{Discussion}

Pericarditis has become recognized as an important cause of morbidity in patients on a chronic, के intermittent haemodialysis regime. Barber et al. (1963) found evidence of pericarditis in four out of thirteen post-mortem examinations performed after prolonged haemodialysis. The report of regular dialysis treatment in Europe for 1969 gives haemorrhagic pericarditis as the cause of mortality in $6 \%$ of patients dying on intermittent dialysis therapy (Drukker et al., 1969).

Comty et al. (1971) reviewing pericarditis in chronic uraemia found an incidence of $16 \%$ of all $\underset{\perp}{\not}$ patients accepted for haemodialysis. In the present $\underset{\nexists}{ \pm}$ series, pericarditis occurred in $18 \%$ of patients 은 established on haemodialysis, and in these patients it appears to have different features from that in patients with severe uraemia. The pericarditis present in three patients on admission to the unis resolved very rapidly $(1,3$ and 7 days respectively) with the institution of regular haemodialysis, along with the other features of severe uraemia. Pericardia effusion and cardiac tamponade have been observe in this uraemic pericarditis (Symons and Wrong, 1964) but did not occur in these three cases.

The type of pericarditis occurring in patients established on dialysis is usually a febrile illness with constitutional features such as malaise and weight loss and it often persists for several weeks. It frequently follows an infective illness and most often presents with chest pain though sometimes has an asymptomatic onset. During the course of the illness, cardiac failure, arrhythmias, pericardial effusion and cardiac tamponade may develop. Pericardial constriction did not occur in this series but has been reported as occurring after an interval of 16 months (Comty et al., 1971). The pericarditis is most frequent in the first 6 months of haemodialysis but may still occur after 3 years. This form of pericarditis should be considered in any patient developing chest pain or an unexplained fever during haemodialysis.

All patients remain uraemic during the course of chronic haemodialysis but at present there is no method of assessing how severe uraemia needs to be for pericarditis to develop. On clinical grounds the patients with pericarditis on established haemodialysis were not uraemic and Table 1 suggests that biochemically they were not severely uraemic when the pericarditis developed.

It is possible that established chronic dialysis 
modifies the natural history of uraemic pericarditis to produce a distinct form of illness. It is felt, though, that this is a separate disease. The aetiology remains obscure and while infective illnesses predispose to pericarditis there is no evidence that the pericarditis is infective.

When effusions were tapped they were found to be heavily blood-stained. This agrees with the findings of other workers for both uraemic pericarditis (Beaudry et al., 1966) and the pericarditis of dialysis (Comty et al., 1971; Piehl et al., 1971). Heparinization for dialysis is clearly a possible cause for the development of a haemorrhagic effusion. Yudis (1971) reported an effusion occurring during the process of haemodialysis and recommended regional heparinization, though this did not prevent four cases developing tamponade in another series (Comty et al., 1971).

In our series effusions have not been closely related to haemodialysis and one patient proceeded to cardiac tamponade while being controlled by peritoneal dialysis. Nevertheless, we have been reluctant to use haemodialysis when the chest X-ray suggests an effusion is expanding. When cardiac tamponade does occur then pericardial aspiration is indicated though one of our cases with clinical tamponade settled spontaneously over a few days.

Systemic steroids have been used with success in seven of eight patients to relieve pericardial pain and fever (Comty et al., 1971) and pericardial instillation of non-absorbable steroid has been recommended at the time of pericardiocentesis (Buselmeier et al., 1973). In view of the high incidence of precipitating infections we have not felt the use of steroids to be justified.

Follow-up in our series has ranged from 2 months to 2 years (mean, 14 months). No evidence of constrictive pericarditis has been found at follow-up although pericardial adhesions have been noted at autopsy in two cases, long after resolution of the clinical pericarditis, and did not appear to contribute to the cause of death.

\section{Acknowledgments}

I would like to thank Dr A. J. Ralston for permission to publish information about his patients and for his advice. I also thank Professor J. M. Evanson for his helpful criticism.

\section{References}

Bailey, G.L., Hampers, C.L., Hager, E.B. \& Merrill, J.P. (1968) Uraemic pericarditis: clinical features and management. Circulation, 38, 582.

BARber, N.D., NAKamoto, S., MCCoRmaCk, L.J. \& KolfF, W.J. (1963) Pathologic anatomy of 13 patients after prolonged periodic haemodialysis. Transactions. American Society for Artificial Internal Organs, 9, 21.

Beaudry, C., NaKamoto, S. \& KolfF, W.J. (1966) Uraemic pericarditis and cardiac tamponade in chronic renal failure. Annals of Internal Medicine, 64, 990.

Buselmeier, T.J., Simmons, R.L., Najarian, J.S., von Hartitzsch, B., Dietzman, R.H. \& KJellstrand, C.M. (1973) Intractable uraemic pericardial effusion. Proceedings of the European Dialysis and Transplant Association, $10,289$.

Comty, C.M., Cohen, S.L. \& Shapiro, F.L. (1971) Pericarditis in chronic uraemia and its sequels. Annals of Internal Medicine, 75, 1973.

Drukker, W., HaAgsma-Schouten, W.A.G., Alberts, C. \& SPOEK, M.G. (1969) Report on regular dialysis treatment in Europe V-1969. Proceedings of the European Dialysis and Transplant Association. 6, 99.

Piehl, W., Wilbrandt, R., Freyland, M.D., Frotscer, U. \& MESSERSCHMIDT, W. (1971) Herzbeutel tamponade bei terminuler Niereninsuffiziens. (Cardiac tamponade in terminal renal failure). Deutsche medizinische Wochenschrift, 96, 1249.

SymoNS, H.S. \& WRONG, O.M. (1964) Uraemic pericarditis with cardiac tamponade-a report of 4 cases. British Medical Journal, 1, 605.

YUDIs, M. (1971) Chest pain in a haemodialysis patient. Lancet, i, 343. 